\title{
Redefiniendo el trabajo social: nuevos yacimientos de empleo en el envejecimiento. Una propuesta desde la intervención social
}

\section{Redefining social work: new employment sources in aging. A suggestion from social intervention}

\author{
CRISTINA DÍAZ ROMÁN \\ Doctoranda Programa Interuniversitario de Doctorado Cuidados Integrales y Servicios de Salud \\ Universidad de Jaén. Jaén (España). cdiaz@ujaen.es. ORCID: https://orcid.org/0000-0002-0443-5322
}

\section{DEL CARMEN MARTÍN CANO}

Doctora en Trabajo Social y Servicios Sociales. Departamento de Psicología. Área de Trabajo Social y Servicios Sociales Universidad de Jaén. Jaén (España). mmcano@ujaen.es. ORCID: https://orcid.org/0000-0001-9294-9640

\section{YOLANDA $M^{\mathrm{a}}$ DE LA FUENTE ROBLES}

Catedrática en Trabajo Social y Servicios Sociales. Departamento de Psicología. Área de Trabajo Social y Servicios Sociales Universidad de Jaén. Jaén (España). ymfuente@ujaen.es.ORCID: https://orcid.org/0000-0002-2643-0100

\begin{abstract}
Resumen: Nos encontramos en un contexto sociodemográfico en el que aumenta progresivamente el número de personas que necesitan de cuidados, principalmente personas mayores de 65 años, colectivo mayoritario en la actualidad, en España. Ello genera que aparezcan nuevas demandas y expectativas sobre la atención prestada. Esto supone para la intervención social enfrentarse a un gran reto. Hasta el momento, las formas de intervención social y de atención proporcionadas por el sistema, no parecen cubrir con plenitud las necesidades emergentes, fundamentalmente por su escasa adaptación a los nuevos contextos sociales y estilos de vida. Socialmente, figuras profesionales como la del/ la asistente personal suponen una inversión provechosa y sostenible en el contexto de la atención y prestación de servicios, puesto que, desde la praxis profesional del Trabajo Social, así como desde el prisma académico o investigador, supone una apuesta para que las personas en situación de dependencia puedan llevar a cabo su proyecto de vida con libertad e independencia.

Por ello, en este trabajo se pretende, por un lado, visibilizar las necesidades percibidas y expresadas por las personas mayores y/o en situación de dependencia, así como sus demandas y, por otro, definir una nueva figura profesional conceptualizada como Técnico/a de Autonomía Personal y Prevención de la Dependencia (TAP) que dé respuesta a los retos planteados a través de la implementación de las nuevas intervenciones basadas en el paradigma de accesibilidad universal y diseño para todas las personas.
\end{abstract}

Palabras clave: Envejecimiento, autonomía personal, vida independiente, Trabajo Social 


\begin{abstract}
We are in a socio-demographic context in which the number of people in need of care, mainly people over the age of 65 , a majority collective today, in Spain, is increasing progressively. This leads to new demands and expectations about the attention paid. This means that social intervention is facing a great challenge. So far, the forms of social intervention and care provided by the system do not seem to fully meet emerging needs, mainly because of their poor adaptation to new social contexts and lifestyles. Socially professional figures such as that of the personal assistant represent a profitable and sustainable investment in the context of the care and provision of services, since, from the professional practice of Social Work, as well as from the academic prism or researcher, it is a bet so that people in dependency can carry out their life project with freedom and independence. Therefore, this work aims on the one hand to make visible the needs perceived and expressed by the elderly and/or in a situation of dependence, as well as their demands and, on the other hand, to define a new professional figure conceptualized as Technical of Personal Autonomy and Prevention of dependency (TAP) that responds to the challenges posed through the implementation of new interventions based on the paradigm of universal accessibility and design for all people.
\end{abstract}

Key words: Aging, personal autonomy, independent life, Social Work

\section{INTRODUCCIÓN}

La población mundial se encuentra inmersa en un profundo proceso de cambios estructurales. La población global continúa creciendo y las previsiones, realizadas por la Organización de Naciones Unidas (en adelante ONU), en 2019, marcan el mantenimiento de esa tendencia ascendente para los próximos años (ONU, 2019).

Este incremento de la población también se hace notable en España, situándolo como uno de los países más envejecidos del mundo (Abellán, Ayala, Pérez y Pujol, 2018). Las causas principales que han llevado a este envejecimiento poblacional son, por un lado, el aumento de la esperanza de vida, marcado por la mejora en las condiciones sanitarias $y_{\text {, }}$ por otro, el descenso de la natalidad (Abades y Rayón, 2012).

El envejecimiento, sin duda, ha marcado un reto social, afectando de manera directa a aquellas disciplinas, como el Trabajo Social, que deben dar atención a este colectivo mayoritario de personas mayores de 65 años. Este reto viene acompañado de una nueva realidad social, hasta el momento desconocida, que emerge con novedades en las necesidades de las personas y también con una transformación en sus demandas.

El Trabajo Social, es la disciplina encargada de intervenir en esa nueva realidad. Para ello, y como ha ido haciendo a lo largo de toda su historia, debe transformarse, adaptándose a los nuevos contextos sociales. Sin embargo, esta transformación no solo puede estar presente en las intervenciones prácticas, si no que la re-conceptualización del Trabajo Social debe partir desde la formación universitaria.
Parte del cambio de la titulación universitaria de Trabajo Social tuvo lugar con su incorporación al Espacio Europeo de Educación Superior (EEES), creado a partir de la Declaración de Bolonia en 1999 (Ministros Europeos, 1999). Con ello se pasó de una formación muy teórica, a otra mucho más práctica, basada en la adquisición de competencias. Además, este nuevo modelo de enseñanza recogido en el Libro Blanco del Grado en Trabajo Social, creado por la Agencia Nacional de Evaluación de la Calidad y Acreditación (en adelante ANECA), fomentó la diversidad curricular, permitiendo a las universidades potenciar sus fortalezas y la innovación de las materias impartidas (ANECA, 2004).

\section{METODOLOGÍA}

La elección de un tipo de metodología concreta en toda investigación se considera fundamental, ya que de ello puede depender el éxito de la misma. Por ello, tal y como señalaba Ruiz (2012), es imprescindible reflexionar detenidamente la metodología más adecuada, en función de los objetivos planteados.

Cuando se habla de metodología se hace referencia al "modo en que enfocamos los problemas y buscamos las respuestas, a la manera de realizar la investigación. Nuestros supuestos teóricos y perspectivas, y nuestros propósitos, nos llevan a seleccionar una metodología u otra" (Quecedo y Castaño, 2002, p.7).

Para la realización de este artículo, se ha llevado a cabo una revisión bibliográfica con el objetivo de conocer detalladamente y, a través de diversas visiones, cuál es la situación 
actual del objeto de estudio y cómo ha ido evolucionando a lo largo de la historia.

La principal fuente de información han sido los artículos científicos publicados en revistas de impacto, vinculadas con las Ciencias Sociales y el Trabajo Social de manera más concreta. Todos estos textos se han localizado mediante la búsqueda en bases de datos como Scopus, Web of Science, Journal Citation Reports o Dialnet Plus. Con el objetivo de realizar una búsqueda ordenada y acotada, evitando también la saturación y el colapso de información, la búsqueda se hizo mediante la utilización de varias palabras clave como son envejecimiento, intervención social, Trabajo Social, accesibilidad universal, diseño para todas las personas, entre otras.

Como estrategia de búsqueda de información especializada, se emplearon los operadores booleanos en las bases de datos anteriormente mencionadas.

Estos operadores son palabras inglesas, principalmente AND, OR y NOT, que nos permiten relacionar conceptos de búsqueda. Es así posible ampliar la búsqueda, utilizando AND, para unir dos o más criterios de búsqueda; limitarla, empleando OR o excluir conceptos mediante NOT (Bayardo, 2003).

Por otro lado, también se aplicaron filtros en la búsqueda, como el año de publicación, lo que nos ha permitido llegar más rápido a aquellos documentos actuales, pudiendo ampliar la búsqueda a años anteriores.

\section{RESULTADOS}

\subsection{Demandas sociales emergentes en el envejecimiento}

Las realidades sociales de la población, a lo largo de la historia, han sido cambiantes, evolucionando de manera paralela a los acontecimientos surgidos en cada época ( $\mathrm{Pa}$ niagua, Lázaro y Rubio, 2010). Unidas a esta transformación se encuentran las necesidades demandadas por la población que, de igual manera, han ido cambiando. "El Trabajo Social se caracteriza por ser una profesión que ha sabido adaptarse a las necesidades que han ido presentando individuos, grupos y comunidades" (De la Paz, Rodríguez y Mercado, 2014, p. 223).

Uno de los cambios más significativos experimentados es el increíble crecimiento que está sufriendo la población mundial y que afecta, en mayor porcentaje, a la población mayor de 65 años (Cardona y Peláez, 2012). Este sector de la población, que comprende a las personas mayores de 65 años, se está convirtiendo en uno de los más importantes y mayoritarios. Ello implica que sea uno de los colectivos con más beneficio de la intervención social por parte del Trabajo Social.

Las condiciones, mejoradas, en la vida de estas personas ha generado que hayan cambiado su estilo de vida, implantando nuevas formas de entender el envejecimiento (Gispert, Ruiz- Ramos, Viciana y Clot- Razquin, 2007). A este fenómeno se le podría asignar el nombre de re-conceptualización de la intervención social. Esto propicia un cambio en lo que hasta ahora se conocía como Ciencias Sociales, que no es más que un proceso complejo y cambiante que, por tanto, requiere de una reformulación constante de las demandas y contextos sociales a los que da respuesta, acorde a su transformación (Estrada, 2011). Cada vez es más probable que, además de vivir más años, se mantengan mejores condiciones de vida y un buen estado de salud. Para poder alcanzar estos resultados hay que llevar a cabo un estilo de vida óptimo, en la medida de lo posible, y mantenerla también durante el envejecimiento. Siguiendo este paradigma, apareció el modelo de envejecimiento activo, definido por la Organización Mundial de la Salud (OMS) como "el proceso de optimización de oportunidades, participación y seguridad, con el fin de mejorar la calidad de vida de las personas a medida que envejecen" $(2015$, p.5).

Además de perseguir un envejecimiento saludable, también es necesario que la última etapa de la vida se pueda vivir con la mayor autonomía posible. Este fue uno de los propósitos recogidos en la definición y denominación de la Ley 39/2006, de 14 de diciembre, de Promoción de la Autonomía Personal y Atención a Personas en Situación de Dependencia (en adelante LAPAD), creada para dar respuestas a las necesidades demandadas por la población mayor y/o con discapacidad. La autonomía personal y la capacidad para tomar decisiones propias sobre la vida, debe ser el principio que rige la intervención desde el Trabajo Social.

De esta manera, la conjugación que generan las nuevas demandas, junto con la innovación proporcionada por los agentes sociales y las entidades, proporciona una mezcla ideal para el avance de la disciplina, a través de la creación de nuevas figuras de intervención en el proceso del envejecimiento poblacional. Estos/as nuevos/as profesionales de la intervención social juegan un papel muy importante, pues de ellos/as depende el buen abordaje de esa realidad que afecta a millones de personas, cuyo nexo común es el respeto por la autonomía personal (Caravaca, 2011). De ahí la necesidad de abordar la situación a través de nuevos enfoques profesionales que, incluso, lleven a la creación de nuevas figuras profesionales dentro de las ya existentes (Sarasola, Moya y Pérez, 2014). 


\subsection{La importancia de la autonomía personal}

La definición que la Ley 39/2006, de 14 de diciembre, de Promoción de la Autonomía Personal y Atención a las personas en situación de dependencia propone para la autonomía, establece: "La capacidad de controlar, afrontar y tomar, por propia iniciativa, decisiones personales acerca de cómo vivir de acuerdo con las normas y preferencias propias así como de desarrollar las actividades básicas de la vida diaria" (BOE, n²99, 2006, p. 44144)

La autonomía definida así podría llevar a entenderla como la presencia de una "capacidad para", o por qué no decirlo, de poseer un conjunto de habilidades, aprendizajes, o repertorio conductual, que engrosen esa disposición para poder controlar, afrontar y tomar, por iniciativa propia nuestra acción. Del mismo modo es una capacidad que se configura en torno a uno mismo y a su situación sobre un contexto de actuación que preferentemente intentamos controlar, afrontar y tomar de forma independiente y en función a nuestras propias preferencias para desarrollar las actividades básicas de la vida diaria.

Los nuevos ámbitos de intervención van inexorablemente unidos a los tipos de necesidades vinculados con promoción de la vida independiente, en ese punto concreto es sobre los que actúan los profesionales en la actualidad.

Estas transformaciones, como es el caso de la vida independiente, plantean nuevos desafíos a la intervención social, debido a que ésta permanentemente está enfrentándose con problemas de diversa índole, generados como resultados de transformaciones en las ciencias en que se basa, cambios en la estructura de la sociedad y en la ideología que predomina en ella, lo que conduce a nuevas expectativas en relación con las competencias laborales necesarias para hacer frente a las transformaciones anteriormente señaladas.

Los cambios descritos están dando lugar a situaciones que requieren nuevos modelos de atención social, así como nuevas soluciones y recursos. La actuación de los profesionales en el ámbito social se perfila difícil en la medida en que intervienen en situaciones de riesgo y vulnerabilidad social donde habrá que actuar deprisa, asimilando circunstancias imprevistas y problemas nuevos.

Ello requiere una alta capacidad de análisis de los hechos sociales a partir de los elementos que les transfiere la realidad social. Para este cometido, resultan imprescindibles los instrumentos que una formación superior puede proporcionarle.

\subsection{Modelos de atención en el envejecimiento}

\section{Modelo de Atención Integral Centrada en la Persona (AICP)}

La Fundación Pilares para la Autonomía Personal, defiende el modelo de atención integral y centrada en la persona, dirigido a acrecentar la calidad de vida de las personas en situación de dependencia.

La perspectiva de integralidad requiere tener en cuenta los aspectos biológicos, psicológicos, sociales y medioambientales que nos constituyen como personas, por otro lado, el enfoque de la personalización, esto es centrar la atención en la persona y supone tener en cuenta los deseos, preferencia y participación de la persona usuaria durante todo el proceso de la intervención (Rodríguez, 2013)

Este modelo tiene como objetivo principal conjugar los sistemas y organización de los servicios prestados con las prioridades y deseos de quienes requieren atención o cuidados. La AICP lo que pretende es promover que quien la reciba sea capaz, mediante los apoyos que sean necesarios, minimizar su situación de fragilidad, discapacidad o dependencia y simultáneamente desarrollar al máximo su autonomía personal para de este modo continuar con el desarrollo y control de su proyecto de vida. (Rodríguez, 2013). Comprende unos valores que orientan la atención que se dispensa desde diferentes ámbitos, entre ellos, el reconocimiento de la singularidad de cada persona, su valor como tal, conocer su historia de vida, perseguir que las todas las personas, aunque precisen atención sean capaces de tomar sus propias decisiones y el control de su vida habitual. (Martínez, 2013).

Si bien, no existe un único modelo de AICP, si podemos destacar como característica común el hecho de que mediante estos modelos las personas receptoras de cuidados tienen un rol activo, ya que el objetivo es empoderar a la persona y que pueda ser la protagonista de su vida en todas sus etapas vitales.

La AICP se apoya principalmente en el reconocimiento y valor de la singularidad de cada individuo, y sitúa la mirada en las capacidades y fortalezas, derechos, ofreciendo apoyos personalizados para que todas las personas, con independencia de su estado o características, puedan seguir gestionando, directa o indirectamente, sus propias vidas, incluso aquéllas quienes tienen un importante deterioro. (Martínez, 2017).

\section{Modelo de Envejecimiento Digno y Positivo (EDP)}

La Fundación Ageing Lab es la que defiende la utilización del Modelo de Envejecimiento Digno y Positivo. Un modelo 
que enfrenta el envejecimiento de las personas replanteándose la intervención como un reto que se fundamenta en principios, criterios y el objetivo de fomentar el envejecimiento digno y positivo. No sólo con las respuestas tradicionales y protocolizadas, donde la intervención se realiza a criterio de los profesionales, de su metodología y de las directrices de la organización; sino que el modelo plantea cuales son los ejes de actuación, fruto de una veintena de años de experiencia.

El modelo es definido por la Fundación como la oportunidad que tienen las personas, durante el envejecimiento, de desarrollo vital y como un reto para los profesionales, que tienen la responsabilidad de dar soluciones especializadas que respeten los derechos fundamentales de la persona. En este sentido, el modelo pone a la persona y a su medio ambiente como las claves de la intervención desde la práctica.

El EDP no es un mero marco teórico, sino que hace referencia a un concepto de trabajo y a una forma de hacer en el ámbito de la atención a personas mayores.

Los principios en los que se fundamenta el modelo EDP y que regirá la actuación de la organización y de sus profesionales son:

a. Bioética, que hace referencia a la justicia, la intimidad, la confidencialidad, la autonomía y el empoderamiento

b. Participación Activa, que incluye accesibilidad universal y diseño para todas las personas, apertura a la vida y vínculos afectivos, tecnología e Intergeneracional dad

c. Inteligencia Colaborativa, que se circunscribe en la especialización, el desarrollo humano, el creative commons y el enfoque integral e interdisciplinar;

d. Bienestar, que hace referencia a la prevención, y a la adecuación al ambiente y servicio a la persona, recogiendo entornos físicos, virtuales y sociales; y la

e. Corresponsabilidad, que se centra en la creatividad e innovación, los resultados de mejora continua y la diversidad desde la igualdad.

Asimismo, el modelo EDP considera que la actividad tecnológica debe ser inherente a cada servicio. Una entidad comprometida socialmente incluirá en su modelo de intervención un criterio basado en el uso de la tecnología dentro del propio servicio, así como la facilitación del acceso a la misma a las personas usuarias. Entre sus objetivos estará siempre influir en el progreso social y económico, orientando los resultados del servicio a satisfacer siempre las necesidades manifestadas por las personas usuarias, desde la propia evolución del servicio (Fundación Ageing Lab, 2016).

\subsection{Transformación curricular en la titulación de Trabajo Social}

Este nuevo escenario supone, una más que probable, reorganización de las prestaciones/recursos y también una redefinición de la intervención social.

Por todo ello, resulta necesario reforzar la formación de recursos humanos, organizativos y de gestión, por el desarrollo de la investigación y por consolidar la aportación teórica específica de los estudios con proyección social como disciplina.

La formación universitaria ha ido experimentando grandes cambios a medida que se instauraban normativas educativas en el país (Ginés, 2004). De ellas han dependido la duración de las titulaciones universitarias, las estructuras de las mismas y la carga lectiva que tenían. Sin embargo, el principal cambio en las comunidades universitarias a nivel europeo llegó con la Declaración de Bolonia de 1999. Con esta Declaración se dio comienzo a un proceso largo, impulsado por la Unión Europea, con el que se creó el Espacio Europeo de Educación Superior (EEES). Este cambio tenía como objetivo crear una igualdad en la formación universitaria en el espacio europeo, promoviendo así la movilidad de los/as estudiantes y generando un intercambio de conocimientos (García y Pérez, 2008).

Este acontecimiento supuso una transformación absoluta en la formación que hasta el momento se está llevando a cabo en las universidades, eliminándose también la diferencia entre licenciaturas y diplomaturas. A partir de la entrada en vigor del nuevo plan, sólo existirían grados o dobles grados (Martín y De la Fuente, 2013).

La incertidumbre que se creó en torno a este punto de inflexión, provocó en España que, entidades como la Agencia Nacional de Evaluación de la Calidad y Acreditación (ANECA) realizaran un estudio detallado de la situación encontrada hasta el momento en las distintas titulaciones impartidas en las universidades españolas y recogieran también acciones necesarias para desarrollar en la nueva etapa.

Con respecto a la disciplina de Trabajo Social, se publicó en 2004 el Libro Blanco del Título de Grado en Trabajo Social por la ANECA. Este libro fue el resultado del trabajo de investigación que realizó una red de universidades españolas, en el cual recabaron información relevante sobre la situación actual de la formación y consensuaron el plan a seguir para implementar las nuevas medidas recogidas en la Declaración de Bolonia.

Con este libro se pretendió recoger aquellos aspectos más relevantes que debe tener la titulación y sobre todo, aporta 
pautas específicas para que las universidades españolas puedan adaptar sus planes de estudios a las características marcadas por el Espacio Europeo de Educación Superior. En él se analizaron aspectos fundamentales en los distintos países europeos, como son las entidades que imparten los estudios de Trabajo Social, el número de créditos que tenían asignados estos estudios en los diferentes puntos geográficos, la demanda de la titulación, entre otros. Con este análisis se pudo confirmar que esta titulación está reconocida académicamente en muchos de los países estudiados, ya que en la mayoría esta formación está dentro del ámbito universitario.

Las universidades no exigían unos requisitos básicos para acceder a la titulación, más allá de superar las pruebas básicas de acceso a la misma, que es igualitaria para todas las titulaciones, tradicionalmente conocida en España como selectividad. Otras universidades europeas, además de estas pruebas, valoraban positivamente la relación vocacional del alumnado con la titulación.

En cuanto al análisis de los planes de estudios de las universidades españolas, existía una gran concordancia entre las asignaturas impartidas en las diferentes comunidades autónomas, principalmente entre aquellas asignaturas más tradicionales como son la historia del trabajo social, introducción al trabajo social, sociología, antropología, modelos de intervención en trabajo social, entre otras. Además, el Libro Blanco del Título de Grado en Trabajo Social recoge las competencias, tanto genéricas como específicas que tienen que adquirir el alumnado a lo largo de esta formación universitaria. Todas aquellas universidades que imparten este grado, deben introducir esa adquisición en su enseñanza, para que exista así igualdad, tanto en España como a nivel europeo.

Sin embargo, una vez analizado este Libro Blanco, es posible observar cómo las asignaturas se han ido adaptando a los cambios sociales que han acontecido, introduciendo las nuevas demandas sociales, que son aquellas que pueden facilitar nuevos nichos de trabajo a la profesión. Así lo muestra el estudio llevado a cabo por García-Estañ y Pastor-Seller (2016), en el que se analizaron los planes de estudio del Grado en Trabajo Social de diferentes universidades españolas. Los principales cambios en las asignaturas impartidas aparecieron a partir de la promulgación de la Ley 36/2006, de 14 de diciembre, de Promoción de la Autonomía Personal y Prevención de la Dependencia, profundizando en materia de envejecimiento, discapacidad, situaciones de dependencia y atención a estos colectivos de población.

Siguiendo las palabras de García-Estañ y Pastor-Seller (2016)
"En este contexto es imprescindible que los futuros profesionales del Trabajo Social adquieran competencias, habilidades y conocimientos específicos en materia de dependencia en sus diferentes perspectivas: política, normativa, deontológica, metodología, valoración, prevención, promoción, programación, intervención, evaluación, entre otros" (p.140)

Por ello, alguno de los argumentos que justifican la necesidad del desarrollo de los estudios de grado y posgrado, son los siguientes:

- Permiten completar la formación universitaria, en intervención social ante los nuevos fenómenos de creciente complejidad.

- Posibilitan respuestas a las demandas de formación superior del conjunto de profesionales de la intervención social.

- Favorecen la cobertura de un espacio en el que no existen titulaciones adecuadas a la creciente demanda de titulados superiores en materia de intervención social.

- Permiten equiparar el nivel formativo de los profesionales españoles de la intervención social con los de los demás países desarrollados

Siguiendo esta idea, la Conferencia de Rectores de las Universidades Españolas (en adelante CRUE) confeccionó una serie de libros en los que introducía el diseño para todas las personas en la formación curricular de diferentes titulaciones, entre las que se encuentra Trabajo Social (CRUE y Fundación ONCE, 2014). Esta línea de diseño para todas las personas y la accesibilidad universal no es común encontrarla en los planes de estudios, ya que los conocimientos y las competencias especializadas en esta materia se adquieren con la formación pos-gradual.

Los estudios de postgrado deben jugar un papel fundamental en este escenario; sin ellos no será posible alcanzar los compromisos adquiridos por los Jefes de Estado de los distintos países de la Unión Europea en las diferentes Cumbres celebradas los últimos años, Lisboa, Barcelona, etc. El postgrado es una pieza clave para relacionar el Espacio Europeo de Educación Superior con el Espacio Europeo de Investigación no sólo entre sí, sino también con todas aquellas estructuras que en paralelo van creciendo en sus alrededores con intenciones diversas pero todas ellas diseñadas para al final potenciar la innovación y la competitividad de los sistemas productivos del conjunto. 
En España, actualmente, el referente de formación de posgrado en el ámbito de la accesibilidad se encuentra en la Universidad de Jaén, con el Máster Propio en Accesibilidad para Smart City; La Ciudad Global; y en la Universidad Internacional de Cataluña, con su posgrado en Accesibilidad y Diseño para todos. El primero de ellos, se lleva a cabo en colaboración con Fundación ONCE, y cuenta con un profesorado extenso, más de 40 profesionales que transmiten sus conocimientos a través de las diferentes cápsulas de enseñanza. En su metodología ya incluye los criterios de accesibilidad para conseguir así que este máster pueda llegar a un número mayor de personas, en condiciones de igualdad, ya que los videos están subtitulados, signados y con la imagen del profesorado y de la materia que está impartiendo. El máster, además, es multidisciplinar, ya que acoge perfiles profesionales de todo tipo. Desde que comenzó su andadura en curso académico 2014/2015, ha formado aproximadamente a 250 personas en accesibilidad, de diferentes países del mundo, añadiendo a éstas las 150 personas que ya formó anteriormente con la antigua denominación. En total, 400 personas han recibido formación en esta materia, de las cuales, 50 alumnos/as eran trabajadores/as sociales.

Tal y como señalan Anderson y Wiscott (2003), es fundamental la realización de cambios curriculares para adaptarse a los nuevos tiempos y permitir así que el alumnado tenga mayores conocimientos y oportunidades para implementarlos en puestos de reciente creación o incluso, empleos creados a través de iniciativas de emprendimiento.

\subsection{La respuesta al Design for all, generador de nuevos empleos}

El nuevo paradigma del diseño universal es fundamental para aplicarlo a todos los ámbitos de la vida, ya que su objetivo principal es crear un beneficio global, aportando una mayor calidad de vida y eliminar así la discriminación, adaptándose a la diversidad social.

Este concepto fue definido en el Libro Blanco del Diseño para Todos en la Universidad (2006) como

"la intervención sobre entornos, productos y servicios con el fin de que todos, incluidas las generaciones futuras, independientemente de la edad, el sexo, el género, las capacidades o el bagaje cultural, puedan disfrutar participando en la construcción de nuestra sociedad" (p. 34)

Se considera un concepto transversal que podría afectar positivamente a la profesión de Trabajo Social y a la interven- ción de sus profesionales, por ello, entidades como la Conferencia de Rectores de la Universidad Españolas y otros/as autores/as, están trabajando en este camino. Algunos de los objetivos de este Libro Blanco fueron los siguientes:

1. Establecer una estructura general donde se incluyan los conceptos básicos en Diseño para Todas las Personas e implementarlos en los diferentes centros

2. Introducir aspectos relevantes de este nuevo paradigma en las competencias específicas que deben obtener los/as titulados/as

3. Construir módulos de enseñanza que transmitan los conocimientos del Diseño para Todas las Personas

4. Aportar al alumnado de las herramientas necesarias relacionadas con el Diseño para Todas las Personas para que éste pueda utilizarlas en la intervención social que realicen.

Si se unen todos estos aspectos a los cambios paulatinos que se suceden en la sociedad, no hay otra opción que la evolución de la profesión para adaptarla a los nuevos contextos. Desde hace años, la población mundial está transformándose y envejeciendo a un ritmo rápido, mayoritariamente esté comprendida en una franja de edad superior a los 65 años. A pesar de las mejoras en la calidad de vida de las personas, éstas, a una edad avanzada, suelen presentar situaciones de dependencia, discapacidades, limitaciones de movilidad, etc. Hay que tener en cuenta que este segmento de la población es clave en las intervenciones sociales que se realizarán desde el Trabajo Social, por ello, es fundamental que éstas incluyan el concepto de Diseño para Todas las Personas.

La disciplina de Trabajo Social continúa transformándose y adaptándose a las nuevas realidades sociales, transformación que ha estado presente a lo largo de toda la historia de la profesión (Sobremonte y Rodríguez, 2020). Sin embargo, los nuevos retos sociales no necesitan solo de cambios en la intervención, sino que deben partir desde la formación al alumnado de la titulación de Trabajo Social. Este sería el caso del nuevo paradigma de la accesibilidad universal y el diseño para todas las personas.

Uno de los puntos clave para comenzar esta nueva etapa es, sin duda, la investigación científica por parte de profesorado, egresados/as, alumnado de máster y doctorado, etc. que, posteriormente, irá acompañada de la transferencia del conocimiento, abriendo así posibles nuevos espacios de intervención.

A pesar de los avances que se están produciendo en la unión de estas dos temáticas, el Trabajo Social y el diseño 
para todas las personas, aún queda un largo camino por recorrer para alcanzar las comunidades sostenibles tan nombradas. No se puede pasar por alto que la sostenibilidad está compuesta por tres grandes dimensiones; la económica, la ecológica y la social. Según palabras de Lima, Pastor y Verde (2017), la sostenibilidad es el equilibrio entre el crecimiento económico, el cuidado del medio ambiente y el bienestar social, tareas en las que debemos trabajar.

\subsection{La nueva figura profesional: Técnico/a de Autonomía Personal y Prevención de la Dependencia (TAP)}

En la unión entre dos disciplinas importantes como son el Trabajo Social y la Accesibilidad Universal y Diseño para todas las personas es donde surge el objetivo último de la tesis doctoral que está finalizando. Éste sería la creación de una nueva figura profesional en la intervención social, que parte de/ de la profesional del Trabajo Social y cuya finalidad es mejorar la accesibilidad del entorno donde reside la persona con discapacidad o con dependencia, generando una mayor autonomía personal.

Esta nueva figura parte de las competencias generales y específicas incluidas en la profesión del Trabajo Social, por lo que algunas de las funciones básicas serán las siguientes (ANECA, 1999):

- Capacidad de identificar los factores sociales y psicológicos que intervienen en la situación personal, a nivel individual, grupal, comunitario, etc.

- Detectar conflictos que aparecen a lo largo de la vida

- Responder a situaciones urgentes, de crisis, que puedan surgir, ofreciendo respuestas para solucionarlas y valorando posteriormente las acciones llevadas a cabo

- Promover el crecimiento, desarrollo y autonomía de las personas a las que se atiende

- Valorar las necesidades de las personas para orientar la estrategia que pueda aportarle mayor calidad de vida

- Capacidad para planificar, implementar, revisar y valorar, al final, la práctica llevada a cabo y el cumplimiento de los objetivos marcados al inicio de la intervención

Continuando con esta línea de innovación y teniendo presentes todas las características propias de los/as profesionales del Trabajo Social, se considera imprescindible volver a formular la figura del Trabajador/a Social, añadiendo nuevas competencias de cara a su intervención con personas ma- yores de 65 años y población con discapacidad o diversidad funcional.

Una de las características fundamentales de esta nueva figura propuesta y de todos aquellos nuevos perfiles de intervención social, debe ser el respeto máximo a la autonomía personal, así como a su autodeterminación. Por ello, las actuaciones deben enfocarse en dos planos; el micro-social, que incluye al individuo y sus circunstancias, y el macro-social, que incluye el entorno en el que interacciona la persona.

La resiliencia y el desarrollo personal son dos de los principios rectores de la práctica profesional de la nueva figura, persiguiendo así el desarrollo pleno de las capacidades de la persona, en la medida de lo posible. Con ello, es posible contrarrestas, en gran parte, las actuaciones asistencialistas llevadas a cabo, hasta el momento, por la Ley 39/2006, de 14 de diciembre, de Promoción de la Autonomía Personal y Atención a Personas en Situación de Dependencia (en adelante LAPAD).

Con perfiles de este tipo, es posible potenciar y desarrollar la primera parte que engloba la LAPAD, diseñada para potenciar la autonomía personal y prevenir la aparición de la dependencia.

\section{DISCUSIÓN}

Los datos estadísticos sobre población mundial muestran una transformación enorme en las pirámides de población. La población mayor de 65 años no para de crecer y mantiene su aumento previsto para los próximos años. En España, la población mayor de 65 años supone el 19\% de la población, lo que convierte a este grupo de la población en uno de los más voluminosos. La sociedad tiende a hacer dos lecturas de estos datos, totalmente opuestas. Por un lado, la lectura positiva señala la satisfacción por haber logrado aumentar la esperanza de vida de las personas, mejorando, por tanto, sus condiciones de vida. Y, por otro lado, la lectura negativa hace referencia a la cantidad de recursos que necesita esta población, pudiendo encontrar escasez en ellos y por ello, llegar a una situación de incapacidad para prestar la atención necesaria.

Desde la disciplina de Trabajo Social se realza el gran reto social que supone el envejecimiento y la gran cantidad de oportunidades que aporta, tanto a la profesión como al resto de personas.

Como se ha analizado a lo largo del artículo, la población mayor ha generado cambios importantes en su estilo de vida y ello ha provocado que sus demandas y necesidades sean diferentes. 
Es por ello que se considera de rigor que la profesión del Trabajo Social experimente una re-conceptualización en general y más concretamente desde sus profesionales para adaptar sus intervenciones a los nuevos contextos sociales.

\section{CONCLUSIONES}

Tras observar el estado actual de la disciplina, es posible concluir la importancia de crear nuevas figuras profesionales que sean capaces de dar respuesta a aquellas demandas o necesidades descubiertas.

En el empeño de transformar la atención prestada desde el Trabajo Social, es importante resaltar la relevancia de la nueva figura profesional propuesta, conceptualizada como Técnico/a de Autonomía Personal y Prevención de la Dependencia (en adelante TAP), en su interacción con la población mayor, personas con discapacidad y/o en situación de dependencia.

Para esta revisión, se han encontrado limitaciones referentes a la dificultad para hallar documentos científicos relacionados con el problema de investigación. Es cierto que existen numerosos documentos sobre envejecimiento, discapacidad, accesibilidad, etc. Sin embargo, apenas aparecen documentos que relacionen todos los conceptos. Tampoco se han encontrado evidencias de la existencia de figuras profesionales como las del TAP. Por todo ello, es posible confirmar la necesidad que existe de crear esta figura, que se constituye como referente en materia de accesibilidad universal y diseño para todas las personas. Sus funciones van encaminadas a proporcionar asesoramiento personalizado sobre productos de apoyo, servicios, prestaciones o estrategias para mejorar la calidad de vida de las personas usuarias, así como el estudio del hogar y su entorno en materia de accesibilidad.

La accesibilidad se ha convertido en la herramienta principal para proporcionar la autonomía personal y evitar, en la medida de lo posible, la aparición de situaciones de dependencia.

\section{BIBLIOGRAFÍA}

Abades, M. y Rayón, E. (2012). El envejecimiento en España: ¿un reto o problema social? Gerokomos, 23 (4), 151 - 155. http://dx.doi.org/10.4321/S1134-928X2012000400002

Abellán, A., Ayala, A., Pérez, J. y Pujol, R. (2018). Un perfil de las personas mayores en España, 2018. Indicadores estadísticos básicos. Informes Envejecimiento en red 17. http://hdl.handle.net/10261/164389
Agencia Nacional de Evaluación de la Calidad y Acreditación (ANECA) (2004). Libro Blanco Título de Grado en Trabajo Social. ANECA

Anderson, D. y Wiscott, R. (2003). Comparing Social Work and Non- Social Work Students' Attitudes About Aging. Implications to promote work with elders. Journal of Gerontological Social Work, 42 (2), 22 - 36. https://doi. org/10.1300/J083v42n02_03

Aragall, F., Benenti, B., Ferrer, J., Fort, J.M., Hernández, J., Merodio, J.R., Monguet, J.M. y Pol, E. (2006). Libro Blanco de Diseño para Todos en la Universidad. Fundación ONCE e IMSERSO. http://riberdis.cedd. net/handle/11181/3445

Bayardo, V. (2003). Rápida y pertinente búsqueda por internet mediante operadores booleanos. Universitas Scientiarum, 8, 51 - 54. https://revistas.javeriana.edu.co/index. $\mathrm{php} / \mathrm{scientarium/article/view/4856}$

Caravaca, C. (2016). Los nuevos ámbitos de intervención desde el ejercicio libre en Trabajo Social. La Razón Histórica, 33, 181 - 202. https://dialnet.unirioja.es/servlet/ articulo? codigo $=6328763$

Cardona, M.D. y Peláez, E, (2012). Envejecimiento poblacional en el siglo XXI: retos y preocupaciones. Revista Científica Salud Uninorte, 28 (2), 335 - 348. http:// rcientificas.uninorte.edu.co/index.php/salud/article/ view/1570/3040

Conferencia de Rectores de las Universidades Españolas y Fundación ONCE (2014). Formación curricular en diseño para todas las personas. Trabajo Social. CRUE. https://biblioteca.fundaciononce.es/publicaciones/colecciones-propias/coleccion-accesibilidad/formacion-curricular-en-diseno-para-4

De la Paz, P., Rodríguez, V. y Mercado, E. (2014). Nuevas tendencias de intervención Trabajo Social. Azarbe, Revista Internacional de Trabajo Social y Bienestar, 3, 223 - 228. https://revistas.um.es/azarbe/article/view/198671/161841

Estrada, V.M. (2011). Trabajo Social, intervención en lo social y nuevos contextos. Prospectiva: Revista de Trabajo Social e Intervención Social, 16, 21 - 53. https://dialnet.unirioja. es/servlet/articulo?codigo $=5857499$

Fundación Ageing Lab (2016). Modelo de Envejecimiento Digno y Positivo (EDP). Cuaderno 0. Cuidados. Fundación Ageing Lab. https://www.sociosanitario.info/publicaciones.html

García, J. (2014). ¿Por qué vivimos más? Descomposición por causa de la esperanza de vida española de 1980 a 2009. Reis. Revista Española de Investigación Social, 48, 39 60. https://doi.org/10.5477/cis/reis.148.39 
García, J.V. y Pérez, M.C. (2008). Espacio Europeo de Educación Superior, competencias profesionales y empleabilidad. Revista Iberoamericana de Educación, 46 (9), 1 - 12. https://doi.org/10.35362/rie4691886

Ginés, J. (2004). La necesidad del cambio educativo para la sociedad del conocimiento. Revista Iberoamericana de Educación, 35, 13 - 37. https://rieoei.org/historico/documentos/rie35a01.pdf

Gispert, R., Ruíz-Ramos, M. Arán, M., Viciana, F. y ClotRazquin, G. (2007). Diferencias en la esperanza de vida libre de discapacidad por sexo y Comunidades Autónomas en España. Revista Española de Salud Pública, 81 (2), 155 - 165. http://doi.org/10.1590/\$113557272007000200006

Ley 39/2006, de 14 de diciembre, de Promoción de la Autonomía Personal y Atención a Personas en Situación de Dependencia (BOE $n^{\circ}$ 299, de 15 de diciembre de 2006). https://www.boe.es/buscar/doc.php?id=BOE-A-2006-21990

Lima, A., Pastor-Seller, E. y Verde, C. (Coords.) (2017). El Trabajo Social: construyendo comunidades sostenibles. Aranzadi

Martín, M.C. y De la Fuente, Y.M. (2013). Trabajo Social en el Espacio Europeo de Educación Superior: el caso español. Documentos de Trabajo Social, 53, 82 - 90

Martínez, M.T. (2013). La atención centrada en la persona. Algunas claves para avanzar en los servicios gerontológicos. Actas de la Dependencia, 8, 25- 47

Martínez, M.T. (2017). La evaluación de los servicios gerontológicos: un nuevo modelo basado en la Atención Centrada en la Persona. Revista Argentina de Gerontología y Geriatría, 31 (3), 83 - 89. https://www.fundacionpilares.org/modeloyambiente/materiales-utiles/ publicacion/evaluacion-de-los-servicios-gerontologicos-un-nuevo-modelo-basado-en-la-atencion-centrada-en-la-persona/

Ministros Europeos (1999). Declaración de Bolonia. Declaración conjunta de los ministros europeos de educación.
Bolonia. http://www.ehea.info/page-ministerial-conference-bologna-1999

Organización Mundial de la Salud (2015). Informe mundial sobre el envejecimiento y la salud. Organización Mundial de la Salud. https://apps.who.int/iris/bitstream/ handle/10665/186466/9789240694873_spa.pdf;jsessionid=DC99D5CC88AA332E722312B6A5EDEDE5?sequence $=1$

Organización Naciones Unidas (2019, 17 de junio). Creciendo a un ritmo menor, se espera que la población mundial alcanzará 9.700 millones en 2050 y un máximo de caso 11.000 millones alrededor de 2100: informe de la ONU [comunicado de prensa]. https://population.un.org/ wpp/Publications/Files/WPP2019_PressRelease_ES.pdf

Paniagua, R., Lázaro, S. y Rubio, E.M. (2010). Cambios y evolución del Trabajo Social en España en el final del siglo XX. Miscelánea Comillas: Revista de Ciencias Humanas y Sociales, 68 (132), 161 - 184. https://dialnet. unirioja.es/servlet/articulo?codigo $=3223977$

Pastor-Seller, E., \& García-Estañ, A. (2016). Análisis de las competencias y conocimientos en atención a la dependencia en Trabajo Social en España. Ciencias Sociales $Y$ Educación, 5(10), 47-74. https://doi.org/10.22395/csye. v5n10a2

Quecedo, R. y Castaño, C. (2002). Introducción a la metodología de investigación cualitativa. Revista de Psicodidáctica, 14, 5 - 40. https://core.ac.uk/download/ pdf/41583619.pdf

Rodríguez, P. (2013). La atención integral y centrada en la persona. Fundación Pilares.

Ruiz, J.I. (2012). Metodología de la investigación cualitativa. Universidad de Deusto

Sarasola, J.L., Mora, M.M. y Pérez, M. (2014). Nuevos enfoques profesionales para el Trabajo Social. Hekademos: Revista educativa digital, 16, 43 - 50. https://dialnet.unirioja.es/servlet/articulo?codigo $=5238529$

Sobremonte, E. y Rodríguez, A. (2020). El Trabajo Socia en un mundo de transformación. Tirant Humanidades 